\title{
KÄHLER-EINSTEIN SURFACES WITH NONPOSITIVE BISECTIONAL CURVATURE
}

\author{
FANGYANG ZHENG
}

(Communicated by Peter $\mathrm{Li}$ )

\begin{abstract}
In this note we show that, for a Kähler-Einstein surface $M$ with negative Ricci curvature and nonpositive bisectional curvature, if the cotangent bundle of $M$ is not quasi-ample then $M$ is a quotient of the bidisc.
\end{abstract}

\section{INTRODUCTION AND STATEMENT OF RESULT}

Let $(M, g)$ be a compact Kähler-Einstein surface with nonpositive bisectional curvature. Assume $c_{1}<0$ (otherwise $(M, g)$ is flat). Then the following was raised in [SY].

Conjecture. Under the above assumptions, $(M, g)$ is isometric to a locally hermitian symmetric surface, namely, a quotient of the ball $B^{2}$ or the bidisc $D \times D$.

For the ball quotient case, the first partial answer was obtained by Siu and Yang [SY] in 1981. We need some notation to describe their theorem.

Denote by $H_{\min }, H_{\mathrm{av}}$, and $H_{\max }$ the minimum, average, and maximum values of the holomorphic sectional curvature in all directions at $p$ (note that $H_{\mathrm{av}}=\frac{1}{3}$ scalar curvature is a constant). Let $a(p)=H_{\mathrm{av}}-H_{\min }$ and $b(p)=$ $H_{\max }-H_{\mathrm{av}}$. Then for any K-E metric one always has $\frac{1}{2} b(p) \leq a(p) \leq 2 b(p)$. Let $\lambda_{0}=2 /\left(1+3 \sqrt{\frac{6}{11}}\right) \quad(\approx 0.622)$. Their theorem is

Theorem (Siu-Yang). Let $(M, g)$ be a compact Kähler-Einstein surface with nonpositive bisectional curvature and $c_{1}<0$. If, for any $p \in M, a(p) \leq \lambda b(p)$ for some $\lambda<\lambda_{0}$, then $M$ is a ball quotient.

In [P] Polombo improved this result by enlarging $\lambda_{0}$ to $\frac{48}{52} \approx 0.923$. (The results in $[\mathrm{P}]$ are actually more general.)

Note that for the bidisc case $a(p) \equiv 2 b(p)>0$ identically, so it is the other end of the story.

Received by the editors November 11, 1992.

1991 Mathematics Subject Classification. Primary 53C55.

Key words and phrases. Kähler-Einstein, bisectional curvature, quasi-ample.

Research supported by National Science Foundation Grant DMS-91-05185 and Duke University. 
For a compact Kähler manifold $M$, let $\pi: P=\mathbf{P}\left(T_{M}\right) \rightarrow M$ be the projectivized tangent bundle and $L$ the tautological line bundle (such that $\pi_{*}(L)=$ $\Omega_{M}$, the cotangent bundle). Then by definition $\Omega_{M}$ is ample (nef) if the line bundle $L$ is ample. Closely related to this is the following:

Definition. $\Omega_{M}$ is said to be quasi-ample if $L$ is nef and $Y \cdot L^{\operatorname{dim} Y}>0$ for any irreducible subvariety $Y \subseteq P$ with $\pi(Y)=M$.

Note that there are many examples of a compact $\operatorname{Kähler~manifold~}(M, g)$ with nonpositive bisectional curvature such that $c_{1}(M)<0$ but $\Omega_{M}$ is not quasi-ample. In the surface case, the ratios of the two Chern numbers $c_{1}^{2} / c_{2}$ of such surfaces can be any rational number between 1 and 2 (cf. [Z]).

In this note we shall give another partial answer to the conjecture:

Theorem. Let $(M, g)$ be a compact Kähler-Einstein surface with nonpositive bisectional curvature. Suppose $c_{1}<0$ and $M$ is not quasi-ample. Then it is a quotient of the bidisc (hence, $g$ is the canonical metric).

\section{Preliminaries}

In this section we shall analyze the quasi ampleness condition. For our purpose we will only consider the dimension-two case. However, the higherdimensional situations are similar.

We shall always assume that $(M, g)$ is a general type Kähler surface with nonpositive bisectional curvature. Then $c_{1}(M)<0$, as $M$ cannot contain any rational curves.

Denote by $\pi: P=\mathbf{P}\left(T_{M}\right) \rightarrow M$ and $L$ the dual of the tautological line bundle on $P$ (such that $\pi_{*}(L)=\Omega_{M}$ is the cotangent bundle). Next let $\hat{g}$ be the hermitian metric on $L$ induced by $g$, and write $Z_{g}=\{(x,[v]) \in$ $\left.P \mid \exists w \neq 0: R_{v \bar{v} w \bar{w}}=0\right\}$ for the zero locus of the bisectional curvature of $g$. It is the subset where the nonnegative curvature form $c_{1}(L, \hat{g})$ fails to be positive definite. A surface $Y \subseteq P$ will be called horizontal if $\pi(Y)=M$.

Since $c_{1}(L, \hat{g})$ is always positive in the fiber direction of $\pi$, it follows that:

Lemma 1. Let $(M, g)$ be a general type Kähler surface with nonpositive bisectional curvature. If $Y$ is a horizontal surface with $L^{2} \cdot Y=0$, then $Y \subset Z_{g}$.

We shall also need the following lemma. Let $C_{1}, C_{2}$ be the two Chern forms under $g$, and let $c_{1}, c_{2}$ be the Chern classes. The nonpositivity of the bisectional curvature implies that $C_{1}^{2}-C_{2} \geq 0$ on $M$. Write $V=V_{g}=\{x \in$ $\left.M \mid C_{1}^{2}(x)-C_{2}(x)>0\right\} . V$ is not empty if and only if $c_{1}^{2}>c_{2}$.

Lemma 2. For $x \in V$ the set $\pi^{-1}(x) \cap Z_{g}$ consists of at most two points.

Proof. Consider the Nakano tensor $N$ defined by $N(\alpha \otimes \beta, \gamma \otimes \delta)=R_{\alpha \bar{\gamma} \beta \bar{\delta}}$ and extended linearly to $T_{M} \otimes T_{M}$. Since $g$ is Kählerian, $N$ is actually a hermitian bilinear form living in $S^{2} T_{M}$. Since every element in $S^{2} T_{x, M}$ is decomposable, the nonpositivity of bisectional curvature implies that $N \leq 0$. Let $\lambda_{1} \leq \lambda_{2} \leq \lambda_{3} \leq 0$ be the three eigenvalues of $N$. It is straightforward to show that $\lambda_{1} \lambda_{2}+\lambda_{2} \lambda_{3}+\lambda_{3} \lambda_{1}=C_{1}^{2}-C_{2}$. So, for $x \in V, \lambda_{1} \leq \lambda_{2}<0$ at $x$. Hence, there can be at most one pair of directions at $x$ which gives zero bisectional curvature. Q.E.D. 


\section{Proof of the Theorem}

From now on we will assume that $(M, g)$ is a compact Kähler-Einstein surface with $c_{1}<0$ and with nonpositive bisectional curvature. The two Chern numbers of $M$ satisfy $3 c_{2} \geq c_{1}^{2} \geq c_{1}>0$.

Lemma 3. For the above $M, c_{1}^{2}>c_{2}$.

Proof. Assume that $c_{1}^{2}=c_{2}$. Then since the Ricci curvature is everywhere negative, by Theorem A of [YZ], $(M, g)$ is locally isometric to a hypersurface in $\mathbf{C}^{3}$. Since $g$ is Einstein, the theorem of Smyth [S] implies that $M$ is locally symmetric, which contradicts our assumption $c_{1}^{2}=c_{2}$. Q.E.D.

Corollary. If $M$ is not quasi-ample, then for any $x \in V$ there exists a unique pair of directions $[\alpha],[\beta]$ at $x$ such that $R_{\alpha \bar{\alpha} \beta \bar{\beta}}=0$. Moreover, $\alpha$ is perpendicular to $\beta$.

Proof. The existence of such a pair is guaranteed by $\pi\left(Z_{g}\right)=M$ (Lemma 1), while the uniqueness comes from Lemma 2. Now suppose $R_{\alpha \bar{\alpha} \beta \bar{\beta}}=0$. Let $\left[\alpha^{\prime}\right]$ and $\left[\beta^{\prime}\right]$ be the directions at $x$ perpendicular to $[\alpha]$ and $[\beta]$, respectively. The Einstein condition implies that $R_{\alpha^{\prime} \bar{\alpha}^{\prime} \beta^{\prime} \bar{\beta}^{\prime}}=0$. So for $x \in V$ these two pairs must coincide. Hence $\alpha \perp \beta$. Q.E.D.

Now we are ready to prove the theorem stated in $\S 1$.

Proof of the Theorem. Let $Y$ be a horizontal surface with $L^{2} \cdot Y=0$. Then $Y \subseteq Z_{g}$. So by Lemma 2 the degree $d$ of the restriction map $\left.\pi\right|_{Y} \rightarrow M$ is one or two. First let us assume that $d=1$.

Note that the metric $g$ is analytic, so $V$ is an open dense subset of $M$. Since $d=1, Y$ is a blowing up of $M$ at finitely many points $E=\left\{p_{1}, \ldots, p_{r}\right\}$. For any $x \in M \backslash E$ choose a holomorphic tangent frame $\left(e_{1}, e_{2}\right)$ with $\left[e_{1}\right] \in Y$ in a neighborhood of $x$. Let $(\alpha, \beta)$ be a unitary frame near $x$ with $[\alpha]=$ [ $\left.e_{1}\right]$. Now $f=R_{\alpha \bar{\alpha} \alpha \bar{\alpha}} \equiv a$ is globally defined in $M \backslash E$, where $a$ is the Ricci curvature. By the computation in [SY], $\Delta f=\left|R_{\alpha \bar{\beta} \alpha \bar{\beta}}\right|^{2} \quad\left(f\right.$ is $S_{1 \overline{1} 1 \overline{1}}$ in [SY]; here we used the fact $\left.[\alpha]=\left[e_{1}\right]\right)$. So $R_{\alpha \bar{\beta} \alpha \bar{\beta}}=0$. Then it follows that the connection and hence, the holonomy split (note that $g$ is analytic). So $(M, g)$ is reducible. Each de Rham factor of the universal covering space is again Einstein with negative Ricci curvature and hence, the Poincaré disc.

Next let us assume $d=2$. Again let

$$
E=\left\{x \in M \mid \pi^{-1}(x) \subseteq Y\right\} .
$$

$E$ is finite as $Y$ is irreducible and horizontal. Since, for $x \in V,\left(\left.\pi\right|_{Y}\right)^{-1}(x)$ consists of two perpendicular directions and $V$ is dense in $M$, it follows that $\pi \mid Y$ cannot have any branch locus over $M \backslash E$. Hence it gives a 2-sheets unbranched covering over $M \backslash E$ and, hence, an unbranched double cover $M^{\prime} \rightarrow M$. Now by the branched covering trick, in the pullback of the fiber bundle $\pi: P \rightarrow M$ by $M^{\prime} \rightarrow M$, the inverse image of $Y$ consists of two irreducible components, each with degree one over $M^{\prime}$, so we are in the first case (or it gives a holomorphic splitting of $T_{M^{\prime}}$ in $M^{\prime} \backslash E^{\prime}$; therefore, $T_{M^{\prime}}$ is not stable and $\left.M^{\prime}=D \times D / \Gamma\right)$. Q.E.D. 


\section{REFERENCES}

[P] A. Polombo, Condition d'Einstein et courbure négative en dimension 4, C. R. Acad. Sci. Paris Sér. I Math. 307 (1988), 667-670.

[S] B. Smyth, Differential geometry of complex hypersurfaces, Ann. of Math. (2) 85 (1967), 246-266.

[SY] Y.-T. Siu and P. Yang, Compact Kähler-Einstein surfaces of nonpositive bisectional curvature, Invent. Math. 64 (1981), 471-487.

[YZ] S.-T. Yau and F. Zheng, On a borderline class of non-positively curved Kähler manifolds, preprint, 1991.

[Z] F. Zheng, On compact Kähler surfaces with non-positive bisectional curvature, preprint, 1992.

Department of Mathematics, Duke University, Durham, North Carolina 27706

E-mail address: zheng@math.duke.edu 